(P2-74) Does Implementation of Protocols Improve the Red Area Disposition Time of Emergency Department at a Level-1 Trauma Center?

R. Kumar, ${ }^{1}$ K. Shyamla, ${ }^{2}$ S. Bhoi, ${ }^{3}$ T.P. Sinha, ${ }^{1}$ S. Chauban, ${ }^{3}$ G. Adhikari, ${ }^{2}$ G. Sharma ${ }^{3}$

1. Emergency Department, New Delhi, India

2. Emergency Medicine, New Delhi, India

3. Department of Emergency Medicine, Trauma Centre, India

Background: Acute care addresses immediate resuscitation and early disposition to definitive care. Delay in final disposition from the emergency department (ED) affects outcomes in terms of morbidity and mortality. An audit was performed to assess the impact of protocols on red area disposition time.

Methods: An audit of red (resuscitation) area disposition time was performed among patients with compromised airway, breathing, and circulation. The red area disposition time was defined as the time from ED arrival to red area disposition. Preprotocol data from nursing report books were reviewed for ED to operating room (OR), ED to intensive care unit (ICU), and overall disposition time between September 2007 and January 2008. Similar outcomes were documented after implementation of protocols during February to December 2008.

Results: In the pre-protocol period, 992 red area patients were enrolled out of 10,000 ED visits. Out of which 527 (53.1\%) were shifted to the OR and $222(22.3 \%)$ to ICU. The average ED disposition time was 3.5 hours (range 2-5). Similarly, $1797 \mathrm{red}$ area patients were enrolled in the post-protocol period out of 25,928. Of these, 453 (25.2\%) patients were shifted to the OR, and $423(23.7 \%)$ were shifted to the ICU. The average ED disposition time was 1.5 hours (range 10 minutes3 hours).

Conclusions: Implementation of protocols improves the red area disposition time of the ED. Auditing is an important tool to address patient safety issues.

Prehosp Disaster Med 2011;26(Suppl. 1):s160

doi:10.1017/S1049023X11005188

(P2-75) Emergency Medicine Begins with General Practitioners in Nepal

\section{R.K. Mabarjan}

Department of General Practitioner \& Emergency Medicine, Kathmandu, Nepal

Introduction: Emergency medicine (EM) is a long-awaited specialty to be established in Nepal, although it has been practiced for a long time starting with hospitals in Nepal. Currently, almost all hospital emergency departments in Nepal are run under general practitioners (GPs) as emergency consultants (emergency physicians) and medical officers work as 24-hour duty doctors.

Methods: This was a five-year observational study (2006-2010) after the Department of General Practice joined with MD in Tribhuvan University Teaching Hospital, Institute of Medicine, Kathmandu, Nepal.

Results: There are no academically trained MD physicians working in Nepal according to the Nepal Medical Council's Registered Doctors, however, there are Nepalese doctors trained and working in EM in foreign countries. There is a demand of training those GPs interested in EM in a Fellowship/MPhil/ MD program. There is a need for developing curriculum of EM for the Fellowship/MPhil/MD or for a different level of training collaborating with universities abroad. There is no ideal prehospital EM/emergency medical services capable of ambulance services. There must be a national code for mobilization and it must be under an umbrella of a governmental Department of GP and EM. Development of infrastructure for the EM department in every hospital in different parts of country is also a challenging task. Standards must be developed for EM and the EMS according to the need of the country. All known and unknown challenges can be addressed by coordinating with international support.

Prehosp Disaster Med 2011;26(Suppl. 1):s160 doi:10.1017/S1049023X1100519X

(P2-76) Mortality Pattern in the Emergency Department in Tribhuvan University Teaching Hospital, Kathmandu, Nepal

\section{R.K. Maharjan}

Department of General Practitioner \& Emergency Medicine, Kathmandu, Nepal

Introduction: The emergency department (ED) deals with serious diseases, trauma, and terminal stage cases. The mortality pattern of cases in the ED must be assessed for future planning and development.

Objectives: The aim of this study is to evaluate the pattern of the mortality cases in ED.

Methods: Retrospective mortality data were collected and analyzed during April to September 2010 from the Tribhuvan University Teaching Hospital.

Results: There were 81 mortality records found during the study period. The mortality rate among the ED cases was 36 per 10,000 (0.36\%). Sixteen (19.75\%) were dead upon arrival to the ED. Among the remaining 65 mortality cases, 30 (46.2\%) were male and $35(53.8 \%)$ female. The ages ranged from 1 to 80 years; the mean age for males was 43.8 years and 55.0 years for females. The mean time duration from when the patient was bought to the ED to death was 6.7 hours. The primary causes of mortality were hypovolumic with hemorrhagic shock $(10,15.5 \%)$, aspiration pneumonitis $(9,13.8 \%)$, cardiopulmonary arrest $(8,12.4 \%)$, sepsis and septic shock $(7,10.8 \%)$, severe head injury $(6,9.3 \%)$, acute exacerbations of chronic obstructive pulmonary disease $(6,9.3 \%)$, hemorrhagic cerebrovascular accident $(3,4.7 \%)$, hepatic encephalopathy $(3,4.7 \%)$, cardiogenic shock (2,3\%), chronic renal failure (2,3\%), dyselectrolytemia $(2,3 \%)$, anaphylaxis $(1,1.5 \%)$, acute respiratory distress syndrome (1, 1.5\%), meningoencepahalitis $(1,1.5 \%)$, acute myocardial infarction $(1,1.5 \%)$, OP poisoning $(1,1.5 \%)$, pulmonary edema $(1,1.5 \%)$, and severe pneumonia $(1,1.5 \%)$.

Conclusions: The mortality in the ED is due to the high rate of severe and serious cases that arrive at late stages of disease. It also is accounted with severe trauma cases despite vigorous treatment at the ED. The rate also is increased by "Brought Dead" cases which could be reduced with proper emergency medical services.

Prehosp Disaster Med 2011;26(Suppl. 1):s160

doi:10.1017/S1049023X11005206 